\title{
Glass particles produced by laser ablation for ICP-MS measurements
}

Lawrence Berkeley National Laboratory, Berkeley, CA 94720, USA

*Corresponding author. Tel.: +1-510-486-4258; fax: +1-510-483-7303. rerusso@lbl.gov

Jhanis J. Gonzalez, Chunyi Liu, Sy-Bor Wen, Xianglei Mao and Richard E. Russo

\begin{abstract}
Pulsed laser ablation (266nm) was used to generate glass particles from two sets of standard reference materials using femtosecond (150fs) and nanosecond (4ns) laser pulses with identical fluences of $50 \mathrm{~J} \mathrm{~cm}^{-2}$. Scanning electron microscopy (SEM) images of the collected particles revealed that there are more and larger agglomerations of particles produced by nanosecond laser ablation.

In contrast to the earlier findings for metal alloy samples, no correlation between the concentration of major elements and the median particle size was found. When the current data on glass were compared with the metal alloy data, there were clear differences in terms of particle size, crater depth, heat affected zone, and ICP-MS response. For example, glass particles were larger than metal alloy particles, the craters in glass were less deep than craters in metal alloys, and damage to the sample was less pronounced in glass compared to metal alloys samples. The femtosecond laser generated more intense ICP-MS signals compared to nanosecond laser ablation for both types of samples, although glass sample behavior was more similar between ns and fs-laser ablation than for metals alloys.
\end{abstract}

\section{Introduction}

Laser ablation processes depend on many parameters which can be grouped by the source. For example, parameters associated to the laser (wavelength, energy, pulse length, spot size, etc.), the environment surrounding the sample (cell design, gas 
composition, pressure, etc), and the material properties (absorptivity, thermal diffusivity, etc.) are also relevant [1;2]. The total number of parameters that can affect the overall ablation process is too large to be evaluated all at once. Therefore, maintaining some of these parameters fixed is the more efficient way to study their influence on the overall process. For this study, we used the same conditions (table 1) as a previous study on metal particles produced by nanosecond and femtosecond laser ablation, in which the only varied condition was the pulse length.

The present study is focused on glass particles produced from ablation of two series of glass standard reference materials and their characterization by measuring particle size distributions, crater volumes, crater profiles, and establishing correlations with ICP-MS performance. The study of these glass samples is of interest due to their relevance in fields like geochemistry, forensic science, etc; fields in which samples with similar properties (optical absorptivity, thermal diffusivity, etc) are found.

In general, the first step of the ablation process involves the absorption of the laser energy by the sample, followed by the diffusion of the absorbed energy into the sample. After a portion of the laser energy is absorbed, the time to transfer that energy to the sample lattice and to start the removal of the material is approximately 10 ps [3;4]. When this energy is delivered on the nanosecond time scale, the transfer time is sufficient to thermally dissipate that energy into the lattice (glass or metals), as opposed to femtosecond laser pulses. For laser pulses with nanosecond duration, the thermal diffusion of the energy will cause a larger heat affected zone compared to femtosecond laser pulses [5]. This effect will be more pronounced in metals than in glasses due to their higher thermal diffusivity. However, glass samples possess lower absorptivity (higher 
penetration depth) compared to metal samples. Therefore, the energy density experienced by the glass sample during the interaction is smaller than the energy density experienced by metals. The combination of the appropriate conditions (i.e. pulse length and wavelength) which influence these two processes (light absorption and thermal diffusion) can be optimized to improve ablation efficiency.

\section{Experimental}

The experimental system was described in detail elsewhere [6]; in short it included a hybrid femtosecond laser system (150fs), and a Nd:YAG nanosecond (4 ns) laser. An ICP-MS was used to chemically analyze the ablated mass and a DMA (Differential Mobility Analyzer) was used for particle size measurements. The glass samples used in this study were a series of standard reference materials from the National Institute of Standard and Technologies (NIST) and X-ray fluorescence (XRF) monitor samples from Glen Spectra. Table 2 shows the matrix composition for these standards.

\section{Results and discussion}

\section{Particle size measurements}

As in a previous report [6] for metals particles, the number density, particle diameter and span were used to describe the particle size distributions functions.

Throughout these experiments, each sample was ablated at six different surface locations, which resulted in six particle size distribution measurements. The average of these six particle size measurements was calculated and is presented in figure 1 (NIST standards), and figure 2 (XRF standards). The error bars represent the standard deviation of this average. 
Number density: Significant differences in normalized number concentration, (dN/dlogDp) of particles (figures $1 a$ and $2 a$ ) was measured in the 10-400 nm range when using femtosecond compared to nanosecond laser ablation. The larger number concentration particles from nanosecond laser ablation originate from a higher ablation rate (more mass per pulse) figure 3 . Figures $1 b$ and $2 b$, show the particle size distributions normalized to the maximum value. In these two figures, it can be observe that: the closeness of the distributions within a standard series, and the smaller standard deviation between the six repetitions (more reproducible), when using femtosecond laser pulsed ablation compared to nanosecond laser pulsed ablation.

To evaluate if the larger number density of particles observed for nanosecond laser ablation were due to higher ablation rate, three different approaches to determine the volume was used. The first approach consisted of direct measurement of the volume with the white light intereferometric microscope (Zygo, New View 200). Figure 3 shows the crater profiles produced by ns-and fs-laser pulses on NIST 610. These profiles showed that the nanosecond crater is deeper than the femtosecond crater and there was no mass deposited around the craters (no rim formed) as opposed to the case of metals. The net volume ratio (fs/ns) of these craters sections shows that fs-laser ablation produces 0.50 times less ablated volume compared to nanosecond pulsed ablation (table 3). These data support the premise that the larger number density of particles measured using the DMA system for nanosecond laser ablation is associated with the larger amount of mass ablated from the sample.

The second approach used to determine the volume of mass ablated per second involved the calculation using a prism volume equation (as in previous paper [6]). The 
data obtained from this approach are presented in table 4. These data reveals that 875 $\mu \mathrm{m}^{3} / \mathrm{sec}$ and $1625 \mu \mathrm{m}^{3} / \mathrm{sec}$ were ablated by the femtosecond laser and nanosecond laser, respectively. Thus the fs/ns ratio of calculated volume per second was 0.5 as it was from the volume net ratio.

The last approach used to verify fs/ns volume ratios was the use of the number concentration of particles normalized by volume obtained from the DMA. In this case, the DMA number concentration of particles per second reveals that $1598 \mu \mathrm{m}^{3} / \mathrm{sec}$ and $3197 \mu \mathrm{m}^{3} / \mathrm{sec}$ were ablated by the femtosecond laser and nanosecond laser, respectively. This approach also leads to the fs/ns ratio of 0.5 , table 3 .

The breadth of the distributions in term of particle size (Span) was not calculated due to break in the distribution after $380 \mathrm{~nm}$ for both lasers, due to measurements limitations of the DMA system.

Particle diameter: The mode of the particle diameter for the NIST glasses (610616) was $150 \mathrm{~nm}$ and 200 nm for ns and fs pulses, respectively, and 160 and $180 \mathrm{~nm}$ for XRF monitor samples. These data show that nanosecond laser ablation produced slightly smaller particles than femtosecond laser ablation, as was the case of metals (previous paper [6]). The next step was the characterization of particles by means of scanning electron microscopy (SEM). The characterization was carry out to investigate if the difference obtained with the DMA data, showing that the nanosecond pulsed laser produces smaller particles than the femtosecond pulsed laser in the measured range, is due to primary particle size or due to differences in the agglomeration of particles. For SEM measurements, NIST 610 was ablated using the same conditions as above; the ablated mass was collected on clean silicon substrates, figure 4. Particles were collected 
at the exit of the ablation chamber after a distance of 1 meter of tubing (normal input to the ICP-MS). The particle collection time for both nanosecond and femtosecond ablation was the same (480sec)

Figures $4 a-d(n s)$ and $4 e-h(f s)$ show the SEM images of the mass collected from nanosecond and femtosecond laser ablation. Considering that experimental conditions were the same for both lasers and the mass was collected in the same fashion, there is evidence that there are more and larger agglomerates of particles produced by the nanosecond laser compare to the femtosecond laser. However, the primary size of particles does not appear to be significantly different between these two cases, figures $4 d$ $n s$ and $4 h$-fs. In these figures, it is also observed almost exclusively spherical primary particles fro both lasers.

From the ICP point of view, transport efficiency of the large agglomerates could influence the ICP-MS performance in term of precision and accuracy. Considering the case in which the transport of large agglomerates is inefficient due to settling in the chamber or transport tubing, nanosecond laser ablation could generate a lower response compared to femtosecond laser ablation, even though more mass is being ablated. On the other hand, if we consider the case in which these large agglomerations of particles reaches the ICP, there is a strong probability that they will not be completely digested by the plasma; even if they are composed of small primary particles, affecting accuracy due to fractionation and generating spikes in the signal affecting the precision. The ICP-MS response for the matrix element ${ }^{29} \mathrm{Si}$ supports these two premises (figure 5); the femtosecond pulsed laser ablation produces a signal which intensity is 4 times greater 
than the nanosecond pulsed laser ablation case, with fewer spikes in the transient signal (TRSD of 7\% and 3.5\% for nanosecond and femtosecond pulses, respectively).

The mechanisms of soft-agglomerates of different sizes for ns and fs-laser ablation are not understood. Nevertheless, similarities between the primary particle size and morphology from these two lasers suggest that it is unlikely that the observed behavior is due to the laser-material interaction, but rather a post-ablation effect. The nature of these soft-agglomerates, which are assemblies of primary particles held together by weak van der Waals forces [7;8], indicated that they are produced from collision between the solid particles after they are formed. The rate of the collision between primary particles could be affected by increasing the number of particles (more mass as in the nanosecond case), by increasing the collision time (longer plasma cooling time), or by changes in the dynamic expansion patterns of these particles [9].

It has been reported that for particle formation using flame aerosol reactors, the formation of soft-agglomerates starts when particles reach a zone of lower temperature and sintering of primary particles effectively stops[10]. Collision between these primary particles in the region of low temperature leads to the formation of soft-agglomerates. Therefore, increasing or decreasing the time that these primary particles spend in this region (by changing flow rate, flame size, etc) will affect the final size of the softagglomerates [8;10-12]. As parallelism to laser ablation is possible to speculate that the larger size of soft-agglomerates when comparing nanosecond laser ablation to femtosecond laser ablation, could be due to the larger amount of mass ablated by the nanosecond laser, and/or by changes in the dynamic expansion patterns of these particles [9]. 
The ablation behavior, in terms of particle size, crater depth, heat affected zone, and ICP-MS response, of these glasses was completely different from the ablation behavior of metals when comparing nanosecond vs femtosecond lasers. However, it is notable that the ratio of the integrated signal intensity to volume net (table 3) as a measure of the overall improved efficiency is 8 times more efficient for femtosecond compared to nanosecond laser ablation as was the case for the metal samples in which it was shown that there is an improvement of 10, 2.5, and 1.7 times for pure Zn, NIST 627, and NIST 1711, respectively.

Among the differences in the ablation behavior between glass and metal samples are that: glass particles are larger than metal particles, the craters in glass are less deep than craters in metal, and damage (rim formation, debris surrounding the ablated area, etc.) to the sample is less pronounced in glass samples. However, when comparing ns to fs-laser ablation the differences of: particle size, ablated volume, and heat affected zone are much greater for metals than for glasses. For glasses it is notable, in particular, that the rim surrounding the crater is absent, even for nanosecond laser ablation. In general, metal samples could dissipate the energy deeper into the lattice due to their higher thermal diffusivity (a) compared to $\mathrm{SiO}_{2}$, which is the main component of the glass samples used in this study. Table 5 shows a rough estimation of the diffusion length using $L=\sqrt{a^{*} \tau}$, where $\boldsymbol{L}$ is the diffusion length, $\boldsymbol{a}$ is the thermal diffusivity, and $\tau$ is the pulse length. As can be seem, metals possess higher diffusion length compared to $\mathrm{SiO}_{2}$. Therefore, a larger volume of the metal is thermally affected compared to glass samples when ablated with nanosecond laser pulses.

\section{Summary}


The particles produced from nanosecond and femtosecond laser ablation of glass samples showed similar primary sizes and morphology. However, SEM images showed that soft-agglomerates of particles after nanosecond laser ablation are more numerous and larger compared with those from femtosecond laser ablation. This observation could be the result of a larger amount of mass (higher number of particles $/ \mathrm{cm}^{3}$ ) being ablated with nanosecond laser ablation which will increase the number of collision between primary particles, and/or by changes in the dynamic expansion patterns of these particles, as shown in reference [9].

Moreover, even though the nanosecond laser ablated a larger amount of mass the femtosecond laser still produced a higher transient ICP-MS signal ( $\sim 4$ times) and fewer spikes (TRSD of 7\% and 3.5\% for nanosecond and femtosecond pulses, respectively), most likely due to settling of the larger agglomerates during transport and/or the inefficient digestion of the larger agglomerates (ns-laser) by the ICP.

\section{Acknowledgement}

We would like to thank Sotiris E. Pratsinis, for his helpful advice on aerosol particle formation; and Paul Berdahl for productive discussions and for editing the manuscript. This work was supported by the Office of Science, Office of Basic Energy Sciences, Chemical Sciences, Geosciences, and Biosciences Division, and the Deputy Administrator for Defense Nuclear Nonproliferation, Research and Development of the U.S. Department of Energy under Contract No. DE-AC02-05CH11231.

\section{List of tables}

Table 1: Experimental conditions

Table 2: Compositions of the reference standard series NIST 610-616 and XRF's 
Table 3: fs-to-ns volume and response ratios.

Table 4: Volumes calculated from crater geometric shape and from DMA measurements

Table 5: Diffusion length calculated using $L=\sqrt{a^{*} \tau}$. Values for Ak and Zn are included since they were the main components of the alloys studied in reference[6].

\section{List of Figures}

Figure 1: a)NIST 610-616 particle size distribution (DMA) and b) normalized particle size distributions.

Figure 2: a)XRF monitor samples particle size distribution (DMA) and b) normalized particle size distributions.

Figure 3: White light interferometer microscope measurements of the NIST 610 crater profile.

Figure 4: SEM images of NIST 610 particles; a-d) nanosecond $\mathbf{e - h ) ~ f e m t o s e c o n d ~ l a s e r ~}$ ablation.

Figure 5: ${ }^{29} \mathrm{Si}$ transient signals generated by $10 \mu \mathrm{m} / \mathrm{sec}$ ablation (nanosecond and femtosecond)

\section{Reference List}

[1] Gunther D. and Hattendorf B., Trends in Analytical Chemistry 24 (2005) 255265.

[2] Russo R.E., Mao X.L, Liu C.Y, and Gonzalez J., J.Anal.Atom.Spectrom. 19 (2004) 1084-1089. 
[3] K.Sokolowski-Tinten, J.Bialkowski, A.Cavalleri, D.vonderLinde, A.Oparin, J.MeyerterVehn, and S.I.Anisimov, PHYSICAL REVIEW LETTERS 81 (1998) 224-227.

[4] Gonzalez J., Mao X.L, Mao S., and Russo R.E., Journal of Analytical Atomic Spectrometry 17 (2002) 1072-1075.

[5] C.Liu, X.L.Mao, S.S.Mao, X.Zeng, R.Greif, and R.E.Russo, Analytical Chemistry 76 (2004) 379-383.

[6] Gonzalez J., Liu C., Wen S., Mao X., and Russo R.E., Talanta (2007).

[7] Bandyopadhyaya R., Lall A.A., and Friedlander S.K., Powder technology 139 (2004) 193-199.

[8] Tsantilis S. and Pratsinis S.E., Langmuir 20 (2004) 5933-5939.

[9] Koch J., Schlamp S., Rosgen T., Fliegel D., and Gunther D., Spectrochimica Acta Part B-Atomic Spectroscopy 62 (2007) 20-29.

[10] S.E.Pratsinis, Progress in Energy and Combustion Science 24 (1998) 197-219.

[11] Tani T., Takatori K., and Pratsinis S.E., Journal of the american ceramic society 87 (2004) 365-370.

[12] S.E.Pratsinis and S.Vemury, Powder technology 88 (1996) 267-273.

[13] Zeng X., Mao X.L, Greif R., and Russo R.E., Applied Physics A: Materials science and processing 80 (2004) 237-241. 
Table \# 1

\begin{tabular}{|ll|}
\hline * & Conditions \\
- & Argon flow $1.25 \mathrm{Lt} / \mathrm{min}$ \\
- & Laser energy $=\sim 0.25 \mathrm{~mJ}$ \\
- & Fpot size $\sim 25 \mu \mathrm{m}$ \\
- & Scan speed $=10 \mu \mathrm{m} / \mathrm{sec}$ \\
- & Frequency $=10 \mathrm{~Hz}$ \\
- & $\tau_{\mathrm{fs}}=150 \mathrm{fs}$ \\
\hline & $\tau_{\mathrm{ns}}=4 \mathrm{~ns}$ \\
\hline
\end{tabular}


Table \# 2

\begin{tabular}{|c|c|c|c|c|c|c|c|c|c|c|}
\hline SRM & Note & $\mathrm{SiO2}$ & $\mathrm{CaO}$ & $\mathrm{Al2O3}$ & $\mathrm{Na2O}$ & $\mathrm{MgO}$ & MnO & B2O3 & $\mathrm{K} 2 \mathrm{O}$ & Fe203 \\
\hline N610 & Si base glass & 72 & 12 & 2 & 14 & & & & & \\
\hline N612 & Si base glass & 72 & 12 & 2 & 14 & & & & & \\
\hline N614 & Si base glass & 72 & 12 & 2 & 14 & & & & & \\
\hline N616 & Si base glass & 72 & 12 & 2 & 14 & & & & & \\
\hline BR A3 & XRF monitor samples & 37.4 & 0.83 & 14 & 0.13 & 3.2 & 20.8 & 4.2 & 2.16 & 1.16 \\
\hline BR B2 & XRF monitor samples & 41.8 & 21 & 8.6 & 0.09 & 0.23 & 0.89 & & 0.04 & 12.3 \\
\hline BR C3 & XRF monitor samples & 9.9 & 0.03 & 27.1 & 7.9 & & 0.47 & 19.1 & 6.9 & 5.4 \\
\hline BR D2 & XRF monitor samples & 5.3 & 14.2 & 20.6 & 9.6 & 7.4 & & 21.9 & 0.09 & 0.58 \\
\hline BR E2 & XRF monitor samples & 48.9 & 0.6 & 8.5 & 15.3 & & 6.5 & 4 & 0.95 & 0.03 \\
\hline BR F2 & XRF monitor samples & 58.2 & 2.84 & 3 & 1.2 & 0.82 & & 2 & 18.4 & 0.07 \\
\hline
\end{tabular}

Table \# 3

\begin{tabular}{|c|c|}
\hline Sample & NIST 610 \\
\hline Volume net ratio (fs/ns) & 0.50 \\
\hline Total concentrantion $(\mu \mathrm{m} 3 / \mathrm{cm} 3)$ ratio $(\mathbf{f s} / \mathbf{n s})$ & 0.50 \\
\hline Integrated signal intensity ratio (fs/ns) & 4 \\
\hline
\end{tabular}

Table \# 4

\begin{tabular}{|c|c|c|c|c|c|c|c|c|c|c|c|c|c|c|}
\hline & \multicolumn{9}{|c|}{ From crater } & \multicolumn{5}{|c|}{ From DMA } \\
\hline & $\begin{array}{c}\text { Scan speed } \\
\mu \mathrm{m} / \mathrm{sec}\end{array}$ & $\begin{array}{l}\text { Time } \\
\text { (sec) }\end{array}$ & $\begin{array}{l}\text { Distance } \\
(\mu \mathrm{m})\end{array}$ & $\begin{array}{l}\text { Base } \\
(\mu \mathrm{m})\end{array}$ & $\begin{array}{l}\text { High } \\
(\mu \mathrm{m})\end{array}$ & $\begin{array}{c}\text { Area } \\
(\mu \mathrm{m} 2)\end{array}$ & $\begin{array}{c}\text { Volume } \\
(\mu \mathrm{m} 3)\end{array}$ & $\begin{array}{c}\text { Volume/sec } \\
(\mu \mathrm{m} 3 / \mathrm{sec})\end{array}$ & & $\mathrm{nm} 3 / \mathrm{cm} 3$ & $\mu \mathrm{m} 3 / \mathrm{cm} 3$ & $\begin{array}{l}\text { Flowrate } \\
\text { (cm3/sec) }\end{array}$ & $\begin{array}{c}\begin{array}{c}\text { Volume/sec } \\
(\mu \mathrm{m} 3 / \mathrm{sec})\end{array} \\
\end{array}$ & $\begin{array}{c}\text { Volume } \\
\text { fs/ns }\end{array}$ \\
\hline $\begin{array}{r}N 610 \\
\text { fs } \\
\text { fs }\end{array}$ & $\begin{array}{l}10 \\
10\end{array}$ & $\begin{array}{l}170 \\
170\end{array}$ & $\begin{array}{l}1700 \\
1700\end{array}$ & $\begin{array}{l}25 \\
25\end{array}$ & $\begin{array}{c}13 \\
7\end{array}$ & $\begin{array}{c}162.5 \\
87.5\end{array}$ & $\begin{array}{l}276250 \\
148750\end{array}$ & $\begin{array}{c}1625 \\
875\end{array}$ & $\begin{array}{l}\text { ns } \\
\text { fs }\end{array}$ & $\begin{array}{l}6.40 \mathrm{E}+11 \\
3.20 \mathrm{E}+11\end{array}$ & $\begin{array}{l}639.5 \\
319.7\end{array}$ & $\begin{array}{l}5 \\
5\end{array}$ & $\begin{array}{l}3197.5 \\
1598.3\end{array}$ & 0.50 \\
\hline
\end{tabular}



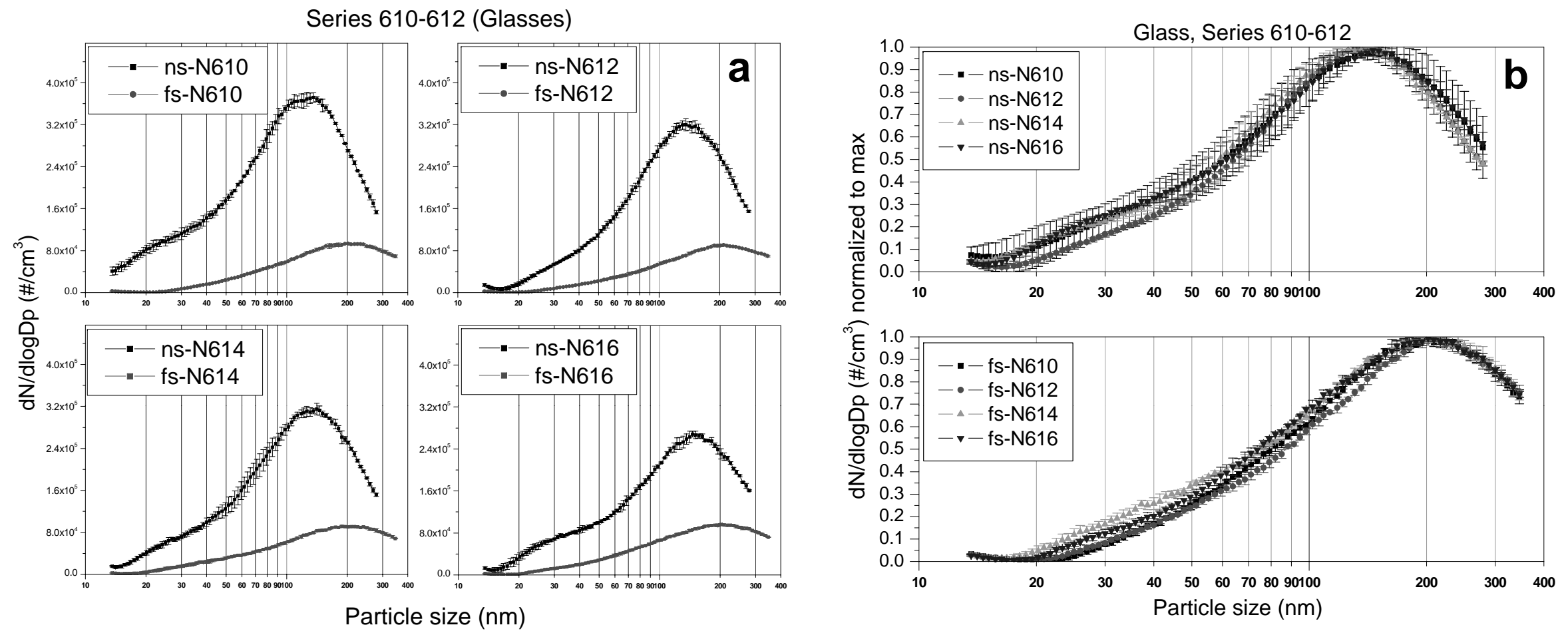

Figure 1 
Series BR-A3-F2 (XRF-Monitor Glasses)
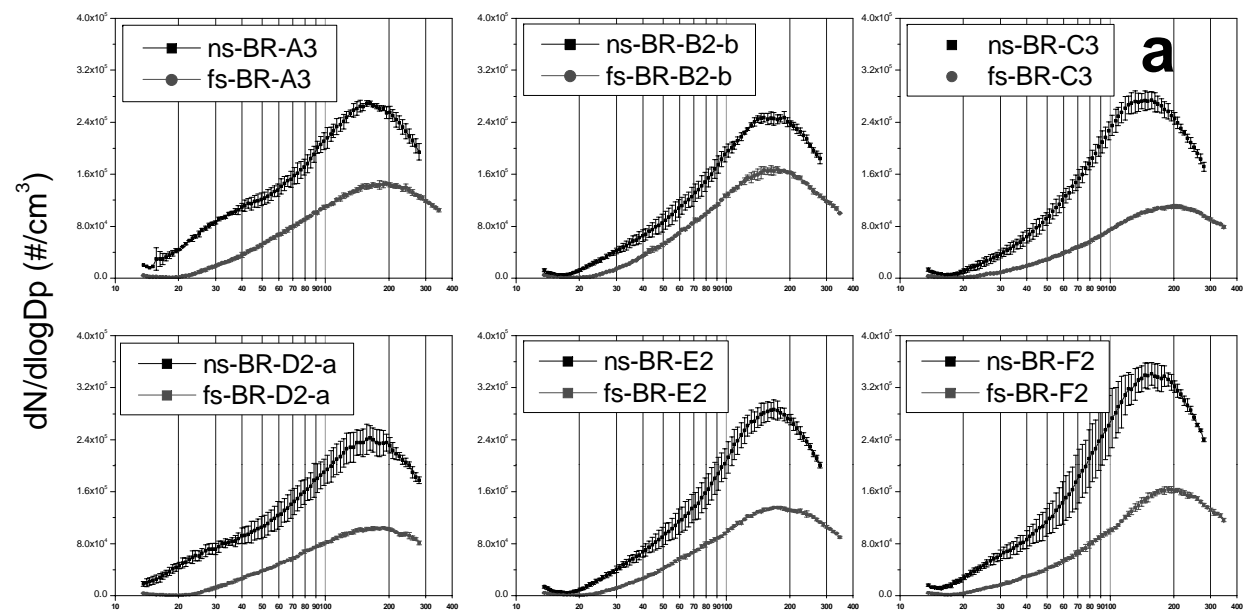

Particle size (nm)
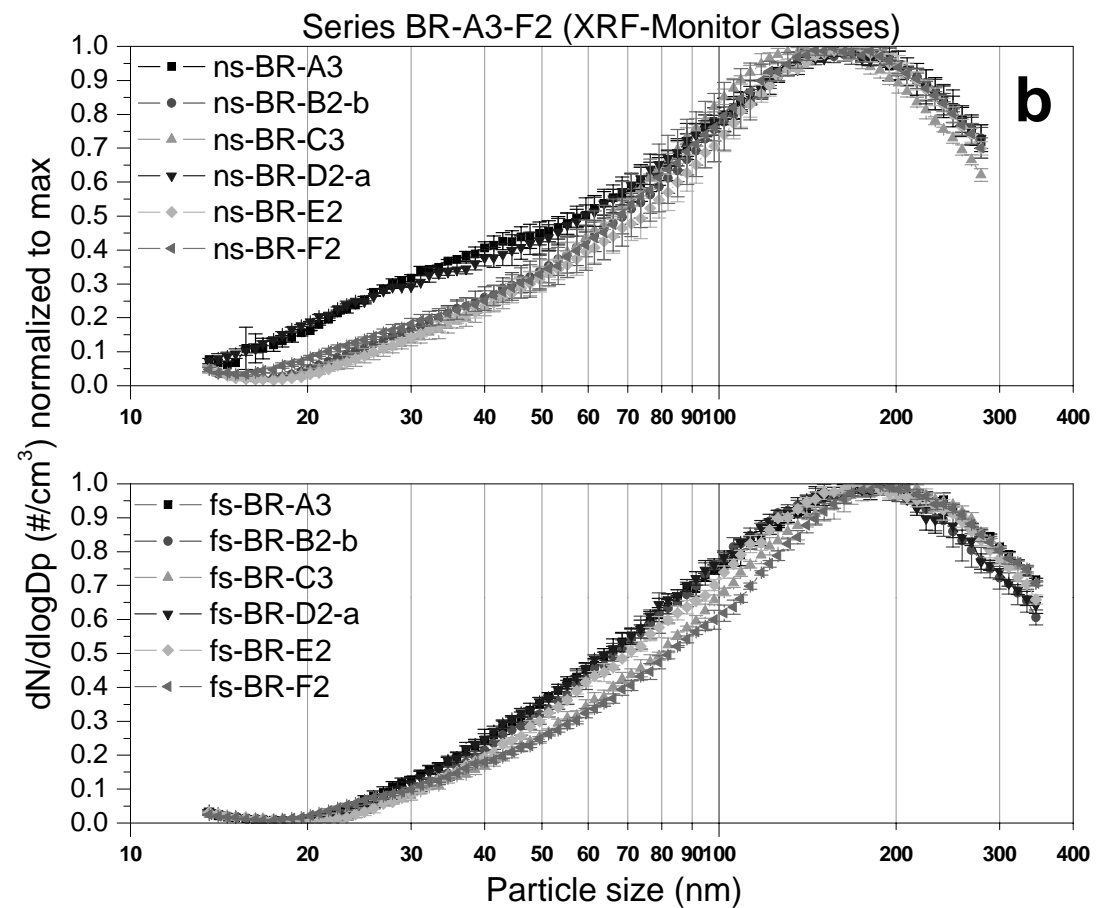

Figure 2 


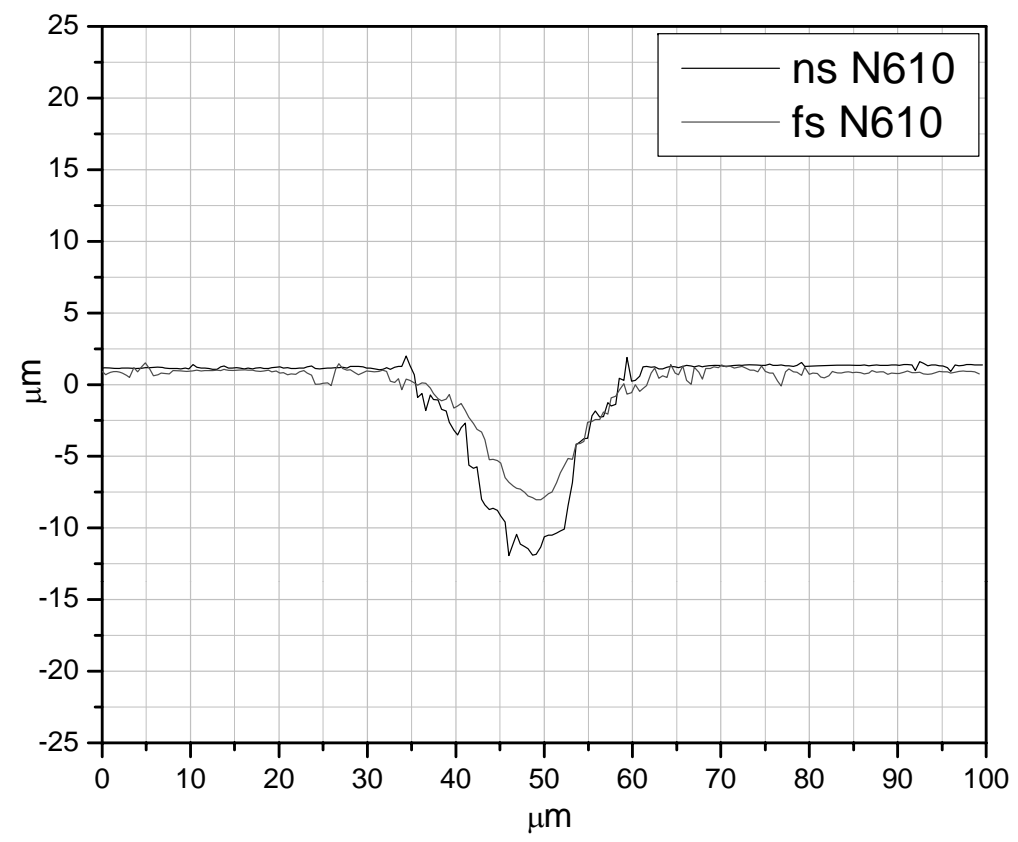

Figure 3 


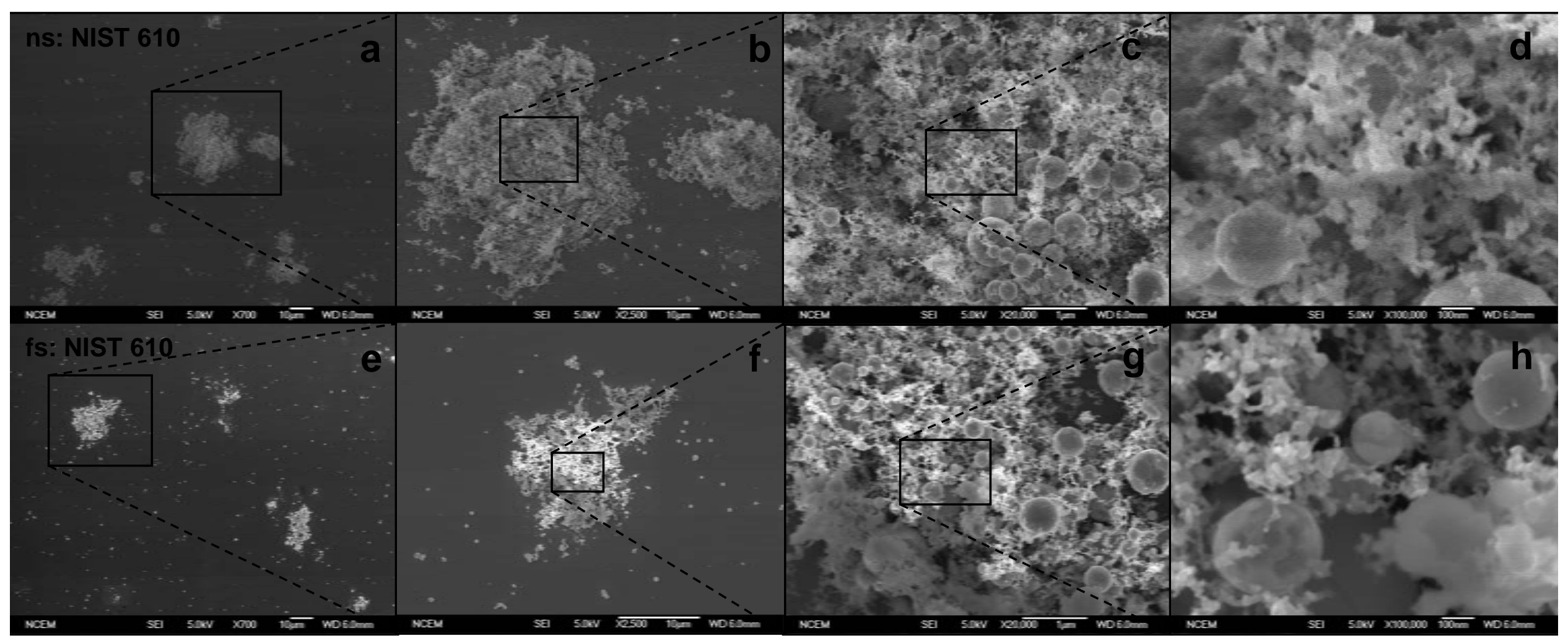

Figure 4 


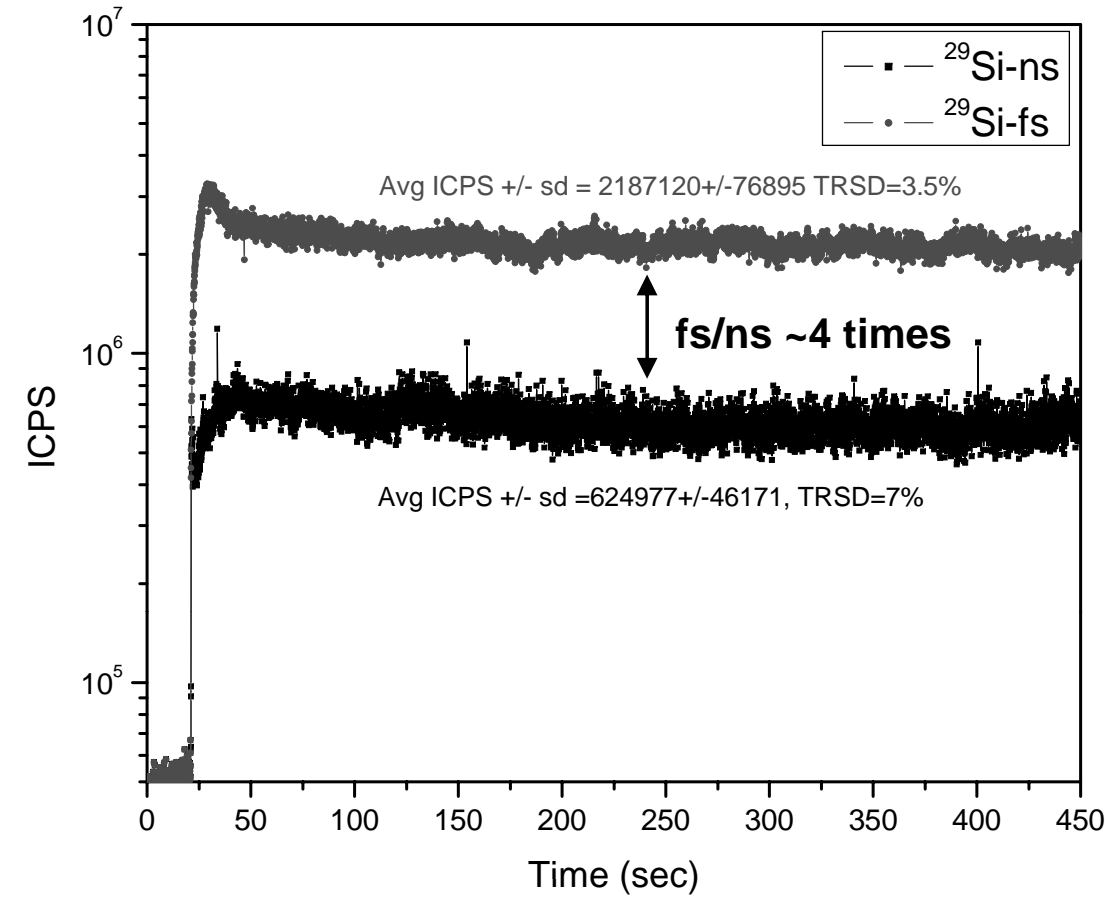

Figure 5 
Table \# 5

\begin{tabular}{|cccccc|}
\hline Element & $\begin{array}{c}\text { Density at } \mathbf{2 5}{ }^{\circ} \mathbf{C} \\
\mathbf{g} / \mathbf{c m} \mathbf{3}\end{array}$ & $\begin{array}{c}\text { Specific heat capacity } \\
\text { J/g K }\end{array}$ & $\begin{array}{c}\text { Thermal conductivity at } \mathbf{2 7} \mathbf{~}^{\circ} \mathbf{C} \\
\text { W/cm K }\end{array}$ & $\begin{array}{c}\text { Thermal diffusivity } \\
\text { m2/s }\end{array}$ & $\begin{array}{c}\text { Diffusion length, } \mathbf{L} \\
\text { nanosecond (nm) } \\
\text { femtosecond (nm) }\end{array}$ \\
\hline $\mathrm{SiO} 2$ & 2.19 & 0.834 & $1.15 \mathrm{E}-02$ & $6.29 \mathrm{E}-09$ & 8 \\
$\mathrm{Al}$ & 2.7 & 0.897 & 2.37 & $8.89 \mathrm{E}-05$ & 0.03 \\
$\mathrm{Zn}$ & 7.14 & 0.388 & 1.16 & $4.18 \mathrm{E}-05$ & 596 \\
\hline
\end{tabular}

